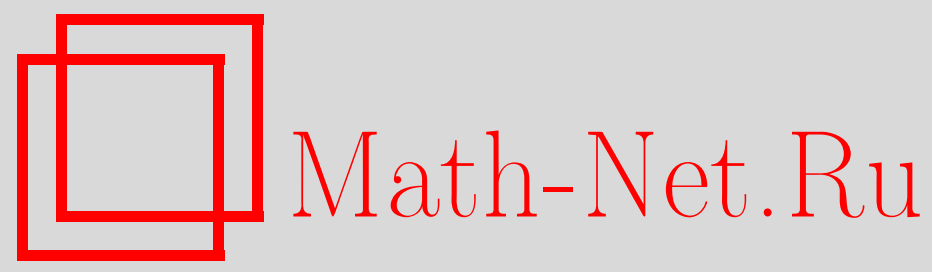

А. В. Лебедев, Максимумы субэкспоненциальных полей дробового шума с конечным радиусом влияния, Матем. заметки, 2003, том 73, выпуск 2, 258-262

DOI: https://doi.org/10.4213/mzm184

Использование Общероссийского математического портала Math-Net.Ru подразумевает, что вы прочитали и согласны с пользовательским соглашением http://www.mathnet.ru/rus/agreement

Параметры загрузки:

IP: 54.198 .67 .100

26 апреля 2023 г., 10:48:45

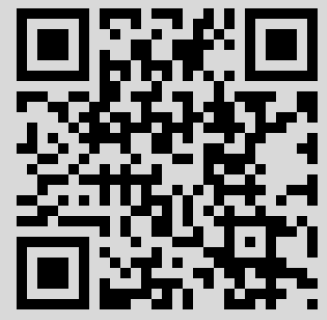




\section{МАКСИМУМЫ СУБЭКСПОНЕНЦИАЛЬНЫХ ПОЛЕЙ ДРОБОВОГО ШУМА С КОНЕЧНЫМ РАДИУСОМ ВЛИЯНИЯ}

\section{А. В. Лебедев}

Изучается асимптотическое поведение максимумов полей дробового шума на ограниченных измеримых областях, стремящимся к бесконечности в смысле Ван Хова. Предполагается, что радиус влияния конечен и амплитуды субэкспоненциально распределены. Получено невырожденное предельное распределение для максимумов.

Библиографоия: 13 названий.

1. Введение. Понятие полей дробового шума возникло из астрофизики в связи с изучением гравитационных полей в звездных системах [1, гл. 4], однако впоследствии оно нашло применение и в других областях (оптике, статистической физике и т.п.). Работы по многомерному дробовому шуму появились в 70-е годы XX века [2], [3] и продолжаются до наших дней [4]-[6]. Заметим также, что решение уравнения Бюргерса со случайными начальными данными [7] в многомерном случае представимо в виде отношения полей дробового шума.

Нашей задачей будет по возможности распространить результаты об асимптотике максимумов субэкспоненциального дробового шума, полученные в одномерном случае [8], на многомерньй. Однако, это удается сделать лишъ за счет ограничений на функцию влияния (в частности, условия финитности).

Рассмотрим евклидово пространство $E=\mathbb{R}^{d}, d \geqslant 1$, и пусть $\left\{\tau_{k}\right\}$ - стационарное пуассоновское точечное поле в $E$ интенсивности $\lambda>0$. Определим формально поле

$$
X(t)=\sum_{k} h_{k}\left(t-\tau_{k}\right), \quad t \in E,
$$

где $h_{k}$ - независимые одинаково распределенные измеримые случайные функции (называемые функииями отклика или влияния [6]), не зависящие от $\left\{\tau_{k}\right\}$.

Величины $h_{k}(0)$ назовем амплитудами, а их распределение обозначим через $F^{0}$. Далее не будем писать нижний индекс при $h_{k}$, если речь идет обо всех $k$.

Предположим, что функции влияния удовлетворяют следующим условиям:

1) $h(t) \geqslant 0$ при всех $t \in E$;

2) $h(t) \leqslant h(0)$ при всех $t \in E$;

3) $h(t)=0$ при $\|t\|>1$;

4) $h(0)<\infty$.

Работа выполнена при финансовой поддержке Российского фонда фундаментальных исследований, грант № 00-01-00131. 
Имеется в виду, что условия 1)-4) выполняются почти наверное (п.н.). Все эти условия обеспечивают существование поля дробового шума $X(t)$ в смысле абсолютной сходимости (1) п.н. [3].

Радиусом влияния естественно назвать величину

$$
\rho=\sup \left\{r \geqslant 0: \mathrm{P}\left(\sup _{\|t\| \geqslant r} h(t)>0\right)>0\right\} .
$$

Каждая точка $\tau_{k}$ вносит вклад в поле $X(t)$ на расстоянии не более $\rho$. Очевидно, значения поля на расстояниях, больших $2 \rho$, независимы между собой. Условие 3 ) означает, что $\rho \leqslant 1$.

Определим теперь для произвольной ограниченной измеримой области $G$ супремум (максимум) поля $X(t)$ по ней:

$$
M(G)=\sup _{t \in G} X(t)
$$

Заметим, что $M(G)$ конечно п.н., так как оценивается сверху суммой амплитуд по всем точкам $\tau_{k}$ в 1 -окрестности области $G$.

Предположим, что задана последовательность таких областей, монотонно стремящаяся к бесконечности в смысле Ван Хова [9, с. 30] (что будем обозначать $G \rightarrow \infty$ ). Напомним это понятие. Рассмотрим разбиение пространства $E$ правильной решеткой из одинаковых прямоугольных параллелепипедов $P_{n}$. Пусть $N^{-}(G)$ - число $P_{n}$, входящих в $G$, и $N^{+}(G)$ - число $P_{n}$, пересекающихся с $G$; тогда стремление $к$ бесконечности в смысле Ван Хова означает, что для любого такого разбиения вьполнено

$$
\lim _{G \rightarrow \infty} N^{+}(G)=+\infty, \quad \lim _{G \rightarrow \infty} \frac{N^{-}(G)}{N^{+}(G)}=1 .
$$

В дальнейшем для простоты вместо произвольных параллелепипедов будем рассматривать только правильные кубы $C_{n}$ со стороной $l$. Например, это могут быть кубы вида

$$
\left[i_{1} l,\left(i_{1}+1\right) l\right] \times \cdots \times\left[i_{d} l,\left(i_{d}+1\right) l\right], \quad i_{1}, \ldots, i_{d} \in \mathbb{Z},
$$

пронумерованные в произвольном порядке.

Обозначим лебегову меру множества $G$ через $|G|$; тогда из (2) следует

$$
N^{-}(G) \sim \frac{|G|}{l^{d}}, \quad N^{+}(G) \sim \frac{|G|}{l^{d}}, \quad G \rightarrow \infty .
$$

Субэкспоненииальные распределения были введены в [10] как распределения, удовлетворяющие следующим условиям:

$$
\begin{aligned}
F(x) & =0, & & x<0, \\
F(x) & <1, & & x>0, \\
\bar{F}^{2 *}(x) \sim 2 \bar{F}(x), & x \rightarrow \infty, & & \text { где } \bar{F}(x)=1-F(x) .
\end{aligned}
$$

Отсюда следует также $\bar{F}^{n *}(x) \sim n \bar{F}(x), x \rightarrow \infty, n \geqslant 2$. Далее класс субэкспоненциальных распределений будем обозначать через $\mathscr{S}$.

Интересные результаты о распределениях из $\mathscr{S}$ были получены в [11], [12]. Далее нам понадобится только одно их свойство, а именно [13]: сумма пуассоновского (со средним $\lambda$ ) числа независимых одинаково распределенных случайных величин с общим распределением $F \in \mathscr{S}$ имеет распределение с хвостом $\bar{F}_{\Pi}(x) \sim \lambda \bar{F}(x), x \rightarrow \infty$. 


\section{2. Основной результат.}

Теорема. Если $\bar{F}^{0} \in \mathscr{S} u и(s)$ - неубьвающая функиия на $(0,+\infty)$ такая, что $s \bar{F}^{0}(u(s)) \rightarrow r \in(0,+\infty)$ npu $s \rightarrow+\infty$, mo

$$
\lim _{G \rightarrow \infty} \mathrm{P}(M(G) \leqslant u(\lambda|G|))=e^{-r}
$$

ДоКАЗАТЕЛЬСТВо. Прежде всего, оценим $M(G)$ снизу максимумом амплитуд по всем $\tau_{k} \in G$ :

$$
\begin{aligned}
\mathrm{P}(M(G) \leqslant u(\lambda|G|)) & \leqslant \mathrm{P}\left(\sup _{\tau_{k} \in G} h_{k}(0) \leqslant u(\lambda|G|)\right) \\
& =\sum_{m=0}^{\infty} \frac{(\lambda|G|)^{m}}{m !} e^{-\lambda|G|}\left(F^{0}(u(\lambda|G|))^{m}\right. \\
& =\exp \left\{-\lambda|G| \bar{F}^{0}(u(\lambda|G|))\right\} \rightarrow e^{-r}, \quad G \rightarrow \infty .
\end{aligned}
$$

Рассмотрим последовательность кубов $C_{n}, n \geqslant 1$ (определенную в п. 1 ), со стороной $l>4$, вместе с концентрическими кубами $C_{n}^{*}$ со стороной $l+2, C_{n}^{\prime}$ со стороной $l-2$ и $C_{n}^{\prime \prime}$ со стороной $l-4$. Оценим $M\left(C_{n}\right)$ сверху:

$$
M\left(C_{n}\right)=\max \left(M\left(C_{n}^{\prime}\right), M\left(C_{n} \cap \bar{C}_{n}^{\prime}\right)\right) \leqslant \max \left\{\xi_{n}, \eta_{n}\right\}
$$

где

$$
\xi_{n}=\sum_{\tau_{k} \in C_{n}} h_{k}(0), \quad \eta_{n}=\sum_{\tau_{k} \in C_{n}^{*} \cap \bar{C}_{n}^{\prime \prime}} h_{k}(0)
$$

Заметим, что $\xi_{n}, n \geqslant 1$, независимы. По свойству субэкспоненциальных распределений имеем

$$
\mathrm{P}\left(\xi_{n}>x\right) \sim \lambda l^{d} \bar{F}^{0}(x), \quad \mathrm{P}\left(\eta_{n}>x\right) \sim \lambda\left((l+2)^{d}-(l-4)^{d}\right) \bar{F}^{0}(x)
$$

при $x \rightarrow+\infty$.

Обозначим через $V^{-}$объединение всех кубов $C_{n}$, входящих в $G$, и через $V^{+}$объединение всех кубов, пересекающихся с $G$. Тогда с учетом (2) получаем

$$
\begin{aligned}
\mathrm{P}(M(G)>u(\lambda|G|)) & \leqslant \mathrm{P}\left(M\left(V^{+}\right)>u\left(\lambda\left|V^{-}\right|\right)\right) \\
& \leqslant 1-\mathrm{P}\left(\xi_{n} \leqslant u\left(\lambda N^{-} l^{d}\right)\right)^{N^{+}}+N^{+} \mathrm{P}\left(\eta_{n}>u\left(\lambda N^{-} l^{d}\right)\right) \\
& \rightarrow 1-e^{-r}+\frac{(l+2)^{d}-(l-4)^{d}}{l^{d}}, \quad G \rightarrow \infty .
\end{aligned}
$$

Переходя в правой части (6) к пределу при $l \rightarrow \infty$ и учитьвая $(5)$, получаем утверждение теоремы. 
ЗАмЕчАниЕ 1. Условие теоремы не требует принадлежности функции $F^{0}$ помимо класса $\mathscr{S}$, еще и области притяжения какого-либо невырожденного максимум-устойчивого распределения при линейной нормировке, как в [8]. В качестве функции $u(s)$ всегда можно взять, например, $u(s)=\inf \left\{x: F^{0}(x) \geqslant 1-r / s\right\}$, пользуясь доказанным еще в [10] свойством: если $F \in \mathscr{S}$, то $\bar{F}(x-y) / \bar{F}(x) \rightarrow 1$ при $x \rightarrow \infty$ для всех $y>0$. В общем случае (4) дает нелинейную нормировку максимумов. Часто нелинейная нормировка может быть сведена к линейной.

ЗАмечаниЕ 2. Теорема показывает, что асимптотическое поведение максимумов не зависит от конкретного вида функции влияния $h$, удовлетворяющей условиям 1)-4), a также от размерности пространства $d$.

ЗАМЕчАнИЕ 3. Величина $\lambda|G|$ инвариантна относительно выбора единиц измерения в пространстве $E$ (как среднее число точек $\tau_{k}$ в области $G$ ). Поэтому теорема остается верна при замене условия 3) на условие вида $h(t)=0$ при $\|t\|>R$ для любого $R>0$, т.е. при любом конечном радиусе влияния $\rho$.

ПримеР 1. Пусть $F^{0}(x)=1-x^{-\alpha}, x \geqslant 1$ (распределение Парето). Тогда $u(s)=$ $(s / r)^{1 / \alpha}$. Полагая $r=x^{-\alpha}$, получаем

$$
\lim _{G \rightarrow \infty} \mathrm{P}\left(M(G) /(\lambda|G|)^{1 / \alpha} \leqslant x\right)=\exp \left\{-x^{-\alpha}\right\}, \quad x>0
$$

ПримеР 2. Пусть $F^{0}(x)=1-\exp \left\{-x^{\beta}\right\}, \beta \in(0,1)$ (распределение Вейбулла). Тогда $u(s)=(\ln (s / r))^{1 / \beta}$. Полагая $r=e^{-x}$, получаем

$$
\lim _{G \rightarrow \infty} \mathrm{P}\left(M(G)^{\beta}-\ln (\lambda|G|) \leqslant x\right)=\exp \left\{-e^{-x}\right\}
$$

Перспективным направлением дальнейших исследований представляется изучение максимумов дробового шума, порожденного точечными полями, более общими, чем пуассоновское (например, ассоциированными [7]), и, возможно, на иных структурах, чем конечномерное евклидово пространство.

Автор благодарен А.В. Булинскому, М.А. Вронскому и рецензенту за внимание к работе, полезные замечания и предложения.

\section{СПИСОК ЦИТИРОВАННОЙ ЛИТЕРАТУРЫ}

[1] Чандрасекар С. Стохастические проблемы в физике и астрономии. М.: ГИИЛ, 1947.

[2] Daley D. J. The definition of multi-dimensional generalization of shot noise // J. Appl. Probab. 1971. V. 8. №1. P. 128-135.

[3] Westcott M. On the existence of a generalized shot-noise process // Stud. Probab. Stat. 1976. P. 74-88.

[4] Булинский А. В. Центральная предельная теорема для полей дробового шума // Проблемы теории вероятн. распределений ХІ. Записки научных семинаров ЛОМИ. Т. 177. Л.: Наука, 1989. С. 28-36.

[5] Gubner J. A. Computation of shot-noise probability distributions and densities // SIAM J. Sci. Comp. 1996. V. 17. № 3. P. 750-761. 
[6] van Lieshout M. N. M., Molchanov I.S. Shot-noise-weighted processes: a new family of spatial point processes // Comm. Stat. Stochastic Models. 1998. T. 14. № 3. C. 715-734.

[7] Бахтин Ю. Ю. Закон повторного логарифма для решения уравнения Бюргерса со случайными начальными данными // Матем. заметки. 1998. Т. 64. №6. С. 812-823.

[8] Лебедев А. В. Экстремумы субэкспоненциального дробового шума // Матем. заметки. 2002. T. 71. № 2. С. 227-231.

[9] Рюэль Д. Статистическая механика. М.: Мир, 1971.

[10] Чистяков В. П. Теорема о суммах положительных случайных величин и ее приложения к ветвящимся случайным процессам // Теория вероятности и ее примен. 1964. Т. 9. № 4. C. $710-718$.

[11] Якымив А. Л. Явные оценки для асимптотики субэкспоненциальных безгранично делимых функций распределения // Матем. заметки. 2000. Т. 67. № 2. С. 295-301.

[12] Балтрунас А. О субэкспоненциальности одного класса случайных величин // Матем. заметки. 2001. Т. 69. №4. С. 625-628.

[13] Embrechts P., Goldie C. M., Veraverbeke N. Subexponentiality and infinite divisibility // Z. Wahr. verw. Geb. 1979. V. 49. № 3. P. 335-347.

Московский государственный университет им. М.В. Ломоносова

Поступило

E-mail: alebedev@mech.math.msu.su

28.08 .2001

Исправленный вариант 10.04.2002 